\title{
An In Vitro Evaluation of Flexural Strength of Two Provisional Restorative Materials Light Polymerised Resin And Autopolymerised Resin.
}

\author{
Surya Prakash Sharma MDS ${ }^{1}$, Ashish.R.Jain MDS $^{2}$, Balasubramanian R MDS ${ }^{3}$ \\ Sathish Alavandar MDS ${ }^{4}$, P.S Manoharan MDS ${ }^{5}$ \\ ${ }^{1}$ Former PG Resident, Department of Prosthodontics, Rajah Muthiah Dental College and Hospitals \\ ,Chidambaram, Tamilnadu, India. \\ ${ }^{2}$ Senior Lecturer, Department of Prosthodontics, Tagore Dental College and Hospitals, Chennai, India. \\ ${ }^{3}$ Professor, Department of Prosthodontics, Rajah Muthiah Dental College and Hospitals, Chidambaram, \\ Tamilnadu, India. \\ ${ }^{4}$ Reader, Department of Prosthodontics, Rajah Muthiah Dental College and Hospitals, Chidambaram, \\ Tamilnadu, India. \\ ${ }^{5}$ Professor, Department of Prosthodontics, Rajah Muthiah Dental College and Hospitals, Chidambaram, \\ Tamilnadu, India.
}

\begin{abstract}
:
Statement of problem: Flexural strength is important in determining the strength of interim restorations and it is especially important in long span and long term interim restorations. Studies comparing the flexural strength of provisional restorative materials are limited and inconclusive.

Aim: To compare the flexural strength of provisional restorative material fabricated using light polymerized composite resin, Urethane dimethacrylate (UDMA) and auto polymerized resin, Poly Methyl Methacrylate (PMMA)

Materials And Methods: This study included 40 specimens. Twenty bar-shaped specimens were fabricated for each group Polymethyl Methacrylate (PMMA), and Urethane dimethacrylate (UDMA) with the use of a split machined metal mold. These specimens were stored in artificial saliva for 10 days. Flexural strength was calculated using three point bending test under universal testing machine. Data were analysed using student-ttest ( $\alpha=0.05) @$ @ 90\% power.

Results: The mean flexural strength of provisional restorative specimen fabricated using Poly Methyl Methacrylate (PMMA-134.1 Mpa) was significantly higher than Flexural strength of provisional restorative specimen fabricated using urethane dimethacrylate (UDMA-107.8 Mpa).

Conclusion: Within the limitations of this study by analyzing the results, it was concluded that the flexural strength of polymethyl methacrylate (PMMA) is comparatively better than the flexural strength of Urethane dimethacrylate (UDMA). PMMA could be a better provisional restorative material for an extended period, when the patient exhibits parafunctional habits, or when long-span prosthesis is planned.

Keywords: Flexural strength, Provisional restoration, Polymethyl methaacrylate (PMMA), Urethane dimethacrylate (UDMA).
\end{abstract}

\section{Introduction}

Provisional restorations also known as interim restoration are critical component of fixed prosthodontic treatment. They are designed to enhance esthetics, stabilization, and function for a short period of time. Patients must be provided with interim restoration from initial tooth preparation until definitive prosthesis is placed. The importance of provisional restoration in fixed dental prosthesis is often overlooked resulting in poorly conceived and fabricated interim restorations. Such restorations will not afford adequate protection and can jeopardize the prepared teeth and supporting tissues. Well made provisional restorations should satisfy biologic and esthetic needs as well as mechanical requirements ${ }^{1,2}$. Mechanically, Interim restorations must be able to withstand the functional forces of mastication without fracture or displacement. This becomes especially important in long term provisional restorations, long span fixed dental prostheses and also during the restorative phase of implant reconstructive procedures. These cases require provisional materials and techniques that provide greater flexural strength and extended durability. Flexural strength is a measurement of the strength of a bar (supported at each end) under a static load. The flexural strength test is a combination of tensile and compressive strength tests and includes elements of proportional limit and elastic modulus measurements. The flexural strengths of interim restorative materials vary within material, chemical classes and between chemical classes of materials. ${ }^{3,45}$ 
Provisional materials have been divided into the following categories based on how they are converted from plastic to solid-elastic masses: (1) chemically activated autopolymerising acrylic resins; (2) heat activated acrylic resins; (3) light-activated acrylic resins; (4) "dual" light and chemically activated acrylic resins; and others (alloys). Presently, there is no provisional material that meets optimal requirements for all situations. Clinicians typically choose a product based on ease of manipulation, cost, and esthetics. When strength is of primary concern, it would be useful to know which materials provide a more resilient provisional restoration. The mechanical properties of a provisional material are also affected by the medium in which it functions. ${ }^{6,7}$

AIM: Therefore a study was designed to compare the flexural strength of polymethyl methacrylate (PMMAAutopolymerizing Resin) and urethane dimethacrylate (UDMA-Light Polymerizing Resin) after storing in artificial saliva.

\section{Materials And Methods}

The protocol of this study was revised and approved by the Department of Prosthodontics, Crown \& Bridge, and Implantology, Rajah Muthiah Dental College and Hospital, Annamalai University, Chennai,India.

\section{Overview of Study Design}

In the context of this study two types of materials resin were selected based on their manipulation and uses. A total of 40 specimens were fabricated (Total Sample size for each group is $n=20$ ). All the specimens were fabricated based on manufacturer's instruction and standardized procedure.

Group A- Autopolymerising resin containing polymethyl methacrylate (PMMA)

Group B- Light polymerising resin containing urethane dimethacrylate. (UDMA)

\section{Statistical Analysis}

Level of significance @ 5\% (0.05)

Power@ 90\%

The following methods of statistical analysis have been used in this study:

1) Student ' $t$ ' test:

The student" $t$ test was used to compare the mean values between the groups.

\section{Fabrication of Metal Framework}

The metal mold was prepared for fabrication of the specimens. The metal mold (Fig 1,2) was made in such a way that it can be split into two halves without distortion of fabricated specimens. The dimensions of the mold were based on American National Standards Institute (ANSI) specification no 27 as $25 \times 2 \times 2 \mathrm{~mm}$. An acrylic plate was fabricated to be kept on the mold so that equal pressure applied on the mold while fabricating the specimens. The metal mold was coated with a layer of petroleum jelly for ease of separation of the specimen from the metal mold after complete polymerisation.

\section{Fabrication of Group "A" Specimen}

The autopolymerising resin (DPI Self Cure Tooth Moulding Powder, Dental Products Of India, Burmah Trading Corporation Ltd, Mumbai, India) powder and liquid were measured and uniformly mixed in the ratio of 2:5 to 1:5. The mixing ratio of powder and liquid was selected based on the findings of Ireland ${ }^{8}$. When the material reaches the dough stage, it was retrieved, kneaded and packed into metal mold and covered by acrylic glass plate over which an equal pressure was applied on plate till complete polymerisation occurs. The material was allowed to set in mold for 15 mins for complete polymerisation. Once completely set, the mold was split carefully and the specimens were retrieved from the mold and examined for porosity or any defect. The excess was removed using tungsten carbide bur and polished. Finally, the PMMA samples were measured using a Digital Vernier Caliper (Digimatic Caliper, Mitutoyo, Japan) for accurate measurement of $25 \times 2 \times 2$ $\mathrm{mm}$. Once it was completed the specimens were immersed in artificial saliva (1L double distilled water, $1.6802 \mathrm{~g}$ $\mathrm{NaHCO}_{3}, 0.41397 \mathrm{~g} \mathrm{NaH} \mathrm{PO}_{4} \cdot \mathrm{H}_{2} \mathrm{O} \& 0.11099 \mathrm{~g} \mathrm{CaCL}_{2}$ ) and stored for 10 days (Fig 3). After 10 days the specimens were taken out, washed and air dried.

\section{Fabrication of Group "B” specimens:}

UDMA material (Ivoclar Vivadent, Inc, Schaan, Liechtenstein) comes in light protected sealed pack. It comes in putty like consistency which can be kneaded, mouldable. The UDMA material was kneaded and packed in metal mold as soon as possible and covered by acrylic glass plate over which uniform load was applied for 2 mins. The mold was then kept in light curing chamber and the specimen initially cured for 2 mins, then the excess material was removed from the sides and again cured for complete polymerisation for 6 mins. 
Finally, the UDMA samples were measured using a Digital Vernier Caliper (Digimatic Caliper, Mitutoyo, Japan) for accurate measurement of $25 \times 2 \times 2 \mathrm{~mm}$. Once it was completed the specimens were immersed in artificial saliva (1L double distilled water, 1.6802g NaHCO $3,0.41397 \mathrm{~g} \mathrm{NaH}_{2} \mathrm{PO}_{4} \cdot \mathrm{H}_{2} \mathrm{O} \& 0.11099 \mathrm{~g} \mathrm{CaCL}_{2}$ ) and stored for 10 days (Fig 4). After 10 days the specimens were taken out, washed and air dried.

\section{Measurement of flexural strength}

The specimens were subjected for testing under universal testing machine (Lloyd's Universal Testing machine, Unitek 94100) which uses 3 point bending test for flexural strength (Fig 5).The specimens were kept on the supports which are $20 \mathrm{~mm}$ apart from each other. The cross head speed of this machine head is $0.75 \mathrm{~mm} / \mathrm{min}$. Each specimen was gradually loaded and the point it flexes and breaks was noted. This is the load required to break the specimen and was obtained in $\mathrm{KN}$ which is converted into Newton. Now the flexural strength was calculated using standard formula,

FLEXURAL STRENGTH $=3 \mathrm{FL} / 2 \mathrm{BH}^{2}$, Where

$\mathrm{F}=$ force / load required to break the samples,

$\mathrm{L}=$ distance between the supports i.e. $20 \mathrm{~mm}$,

$\mathrm{B}=$ width of the specimen i.e. $2 \mathrm{~mm}$,

$\mathrm{H}=$ thickness of specimen i.e. $2 \mathrm{~mm}$

The value obtained for flexural strength is in $\mathrm{n} / \mathrm{m} 2$ which is equal to $1 \mathrm{Mpa}$.

\section{Results}

The differences in flexural strength observed between the provisional restorations fabricated by autopolymerising resin and light polymerized resin were assessed and were subjected to statistical analysis. All data were analyzed by using the statistical program of social science version 18.0(SPSS Inc, Chicago, USA). The data and results of the study are presented in the table (Table I).

TABLE I: Mean, Standard Deviation, Standard Error of Samples Compared and "T" Value, "P" Value Inferences.

\begin{tabular}{|c|c|c|c|c|c|c|c|}
\hline Variables & $\begin{array}{c}\text { Mean } \\
\text { (MPA) }\end{array}$ & $\begin{array}{l}\text { Sample } \\
\text { size }\end{array}$ & $\begin{array}{c}\text { Standard } \\
\text { deviation }\end{array}$ & $\begin{array}{c}\text { Standard } \\
\text { error }\end{array}$ & T value & P value & Inference \\
\hline FS1 & 134.1 & 20 & 17.50 & 3.913 & \multirow{2}{*}{4.96} & 0.0000 & Significant \\
\hline FS2 & 107.8 & 20 & 15.95 & 3.567 & & \\
\hline
\end{tabular}

FS1 - flexural strength of PMMA Samples, FS2 -flexural strength of UDMA Samples

\section{Discussion}

There is minimal consensus cited in the literature regarding criteria for comparision of the flexural strength of polymethyl methacrylate (PMMA) and urethane dimethacrylate (UDMA). Therefore, this study was designed with an aim to compare and evaluate the flexural strength of polymethyl methacrylate (PMMA) and urethane dimethacrylate (UDMA).

Autopolymerising Polymethyl Methacrylate (PMMA) first appeared around 1940 and remains the most frequently used material for fabrication of interim restorations. These have the advantages of good wear resistance, good colour stability, high polishability, and good esthetics. ${ }^{4}$ Although this material meet most of the requirements and is popular even today, its use is on decline because of their disadvantages and development of new and improved materials. The Disadvantages of PMMA have been identified as high level of monomer release, exothermic reaction, and polymerisation shrinkage. ${ }^{9}$

The Visible Light Polymerized (VLC) materials was first introduced in the 1980s, and contained urethane dimethacrylate, a resin whose polymerization is catalyzed by visible light and camphoroquinone as initiator. ${ }^{4}$ UDMA usually incorporate filler such as microfine silica to improve physical properties like reduced polymerization shrinkage. Unlike methacrylate resins they do not produce residual free monomer after polymerization, which explains why they exhibit decreased tissue toxicity. Haddix ${ }^{9}$ indicated that VLC materials could produce provisional restorations with a similar quality but with less time and expense.

The reason for selecting UDMA was that it claims many advantageous properties like

$>$ No MMA, no odour, no exothermic, no irritation.

$>$ Unlimited working time,

Superior handling, easy to place, contour, sculpt and shape.

$>$ Reduced polymerization shrinkage $(0.388 \%)$.

Several Authors have studied the flexural Strength of different provisional restorative materials 
Ireland \& Dixon ${ }^{\mathbf{1 0}}$ tested the modulus of rupture (flexural strength) of four provisional materials and found Bisacryl to have the highest flexural strength. Osman ${ }^{11}$ showed that 2methyl methacrylate provisional materials had higher flexural strength than a composite material. Wang ${ }^{\mathbf{1 2}}$ stated that no significant differences were found between methyl methacrylate and composite provisional materials tested. From the previous studies, there was no proper conclusion that which material has higher flexural strength than other. Therefore in this study the flexural strength of PMMA and UDMA were tested. While flexural strength values obtained in a laboratory under static load may not reflect the conditions found in the oral environment, it is helpful to compare provisional materials tested in a controlled situation. To simulate the oral condition the specimens fabricated from the both materials were immersed in artificial saliva for 10 days ${ }^{13}$.The mean flexural strength of PMMA specimens is $134.1 \mathrm{Mpa}$, and the mean flexural strength of UDMA specimens is $107.8 \mathrm{Mpa}$ which states that mean flexural strength of PMMA specimens is significantly higher than the mean flexural strength of UDMA specimens.

The reason for difference in flexural strength can be; ${ }^{14,15,16}$

> Partly attributed to differences in chemical composition. When combined in different provisional materials, the monomers display differences in properties such as exothermic heat of polymerization and shrinkage resistance.

$>$ During the polymerization, the shrinkage of the resin occurs towards the center of the mass, this may lead to differences in flexural strength. According to the literature, the amount of polymerization shrinkage observed in case of light cured composite resin is about $0.388 \%$, which is significantly less compared to the polymerization shrinkage of PMMA resins, $(6 \%-8 \%)$.

$>$ The UDMA specimens were taken out during initial polymerisation to remove the excess and placed again in the mold for complete polymerisation. This may lead to distortion of specimen and change in flexural strength.

\section{Clinical Implications}

It is important to note that flexural strength is just one of the properties of provisional material. A strong material may possess many other mechanical properties like fatigue strength, impact strength, elastic modulus, fracture resistance, etc and also possess less desirable characteristics such as tendency to stain, lack of polish ability, difficult manipulation, or poor esthetics ${ }^{17}$

\section{Study Limitations}

Based on this study it cannot be state that PMMA is better than UDMA. There are many other properties and different clinical situations which finally choose the provisional material appropriate for each patient.

\section{Future Prospective}

This study can be further improved by inclusion of more types of provisional materials which are available e.g.; heat cure provisional material, bisacryl composite resin, fiber rein forced provisional material etc. This study can also be improved by changing the storage medium of provisional material and also the storage time.

\section{Conclusion}

Within the limitations of this study by analyzing the results, it was concluded that:

$>$ The flexural strength of polymethyl methacrylate (PMMA) is comparatively better than the flexural strength of UDMA

> PMMA could be a better provisional Restorative material for an extended period, when the patient exhibits parafunctional habits, or when long-span prosthesis is planned.

\section{References}

[1]. Francis.B.Robinson\& Howijatra S. Marginal fit of direct temporary crowns. J.Prosthet Dent 1982; 47(4); 390-392.

[2]. Fedrik DR. The provisional fixed partial denture. J Prosthet Dent 1975; 34:520-6

[3]. Fox CW, AbramsBL, \& Doukoudakis. Provisional restoration for altered occlusion. J Prosthet Dent 1984; 52:567-72.

[4]. Michele F. Ireland, Donna L. Dixon. In vitro mechanical property comparison of four resins used for fabrication of provisional fixed restorations. J Prosthet Dent 1998;80:158

[5]. Gegauff AG, Pryor HG. Fracture toughness of provisional resins for fixed. Prosthodontics. J Prosthet Dent 1987; 58:23-9.

[6]. David G. Gratton. Interim restorations. Dent Clin N Am 48 (2004) 487-497.

[7]. Grajower Z, Sharbani S, \& Kaufmane. Temperature rise in pulp chamber during fabrication of temporary self-curing resin crowns. J.prosthet.dent: 1979; 41(5); 535-40.

[8]. Ireland MF, Dixon DL, Breeding LC, Ramp MH. In vitro mechanical property comparison of four resins used for fabrication of provisional fixed restorations. J Prosthet Dent 1998; 80:158 62.

[9]. Haddix JE.A technique for visible light cured provisional restorations.J Prosthet Dent 1988; 59:512-4. 
[10]. Ireland MF, Dixon DL, Breeding LC, Ramp MH. In vitro mechanical property comparison of four resins used for fabrication of provisional fixed restorations. J Prosthet Dent 1998; 80:158 62.

[11]. Osman YI, Owen CP. Flexural strength of provisional restorative materials. J Prosthet Dent 1993; 70:94-6.

[12]. Wang RL, Moore BK, Good acre CJ, Swartz ML, Andres CJ. A comparison Of resins for fabricating provisional fixed restorations. Int J Prosthodont 1989; 2:173-84.

[13]. Hazelton LR \& BrudwikJ. A new procedure to reinforce fixed provisional restorations. J.Prosthet Dent: 1995; 74(1): 110-113.

[14]. Hazelton DR, Diaz-Arnold AM, Vargas MA Flexural strength of provisional crown and fixed partial denture resins J Prosthet Dent 2002;87:225-8.)

[15]. Henry M. Young, Charles T. Smith, Dean Morton, Comparative in vitro evaluation of two provisional restorative materials (J Prosthet Dent 2001; 85:129-32.)

[16]. Hernandez EP, Oshida Y, Platt JA, Andres CJ, BARCO MT\&, Brown DT Mechanical properties of four methylmethacrylate-based resins for provisional fixed restorations. Biomed Mater Eng. 2004; 14(1): 107-22.

[17]. Hunter AJ\& Hunter AR. Gingival margins for crowns: a review and discussion part II discrepancies \& configuration. J.Prosthet Dent 1990; 64: 636-42.

\section{FIGURES}

Figure 1: Metal Mold

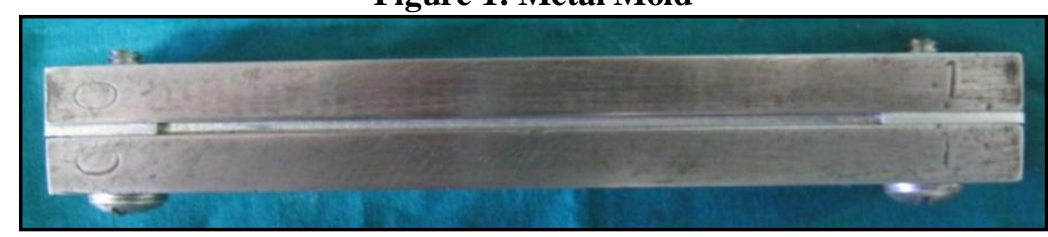

Figure 2: Split Machined Metal Mold

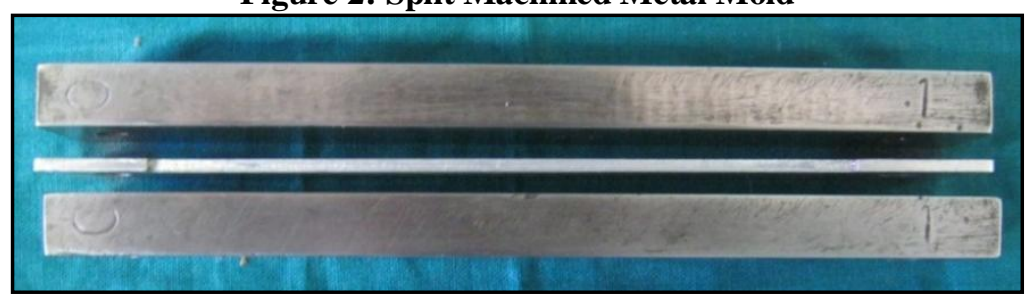

Figure 3: PMMA Samples

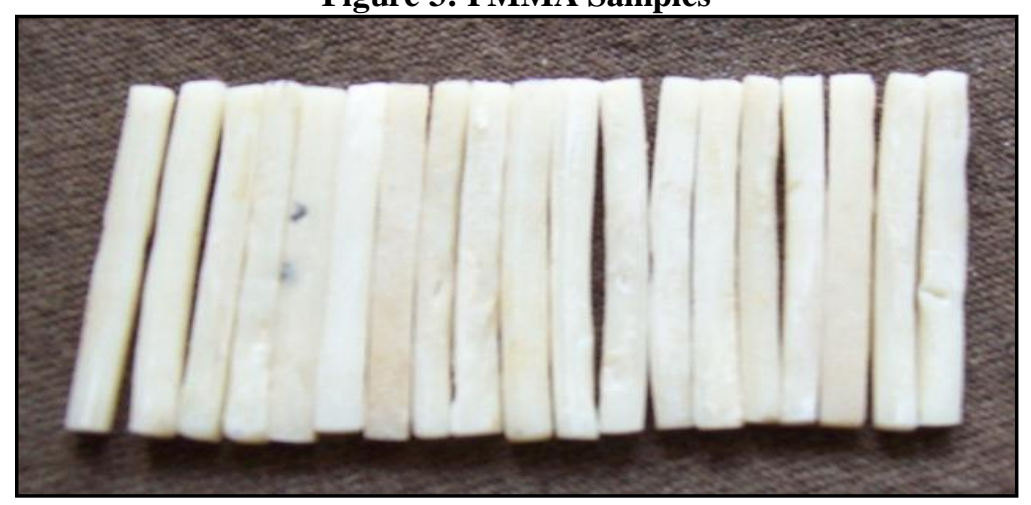

Figure 4: UDMA Samples

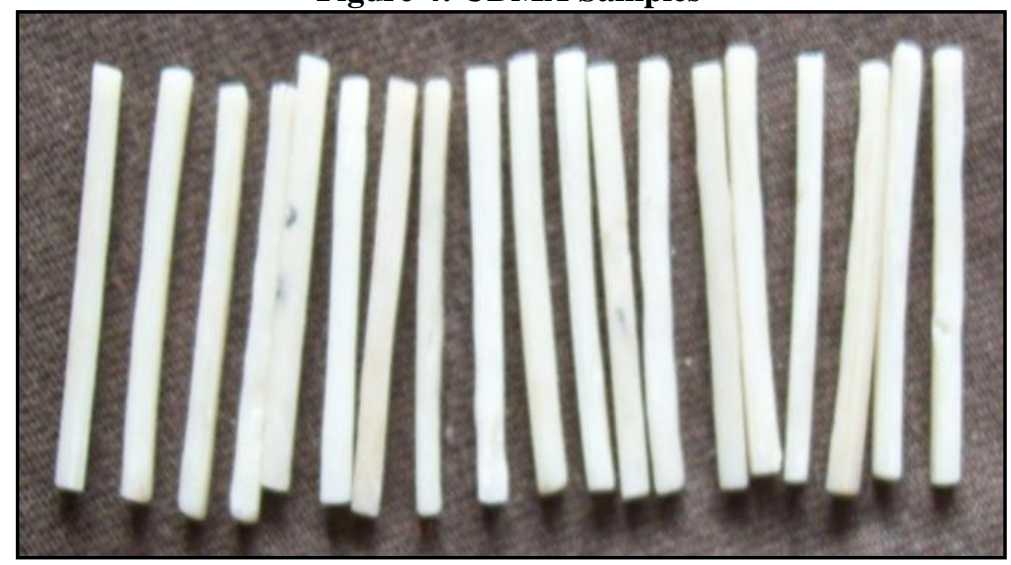




\section{Figure 5: Three Point Bending Test for Flexural Strength}

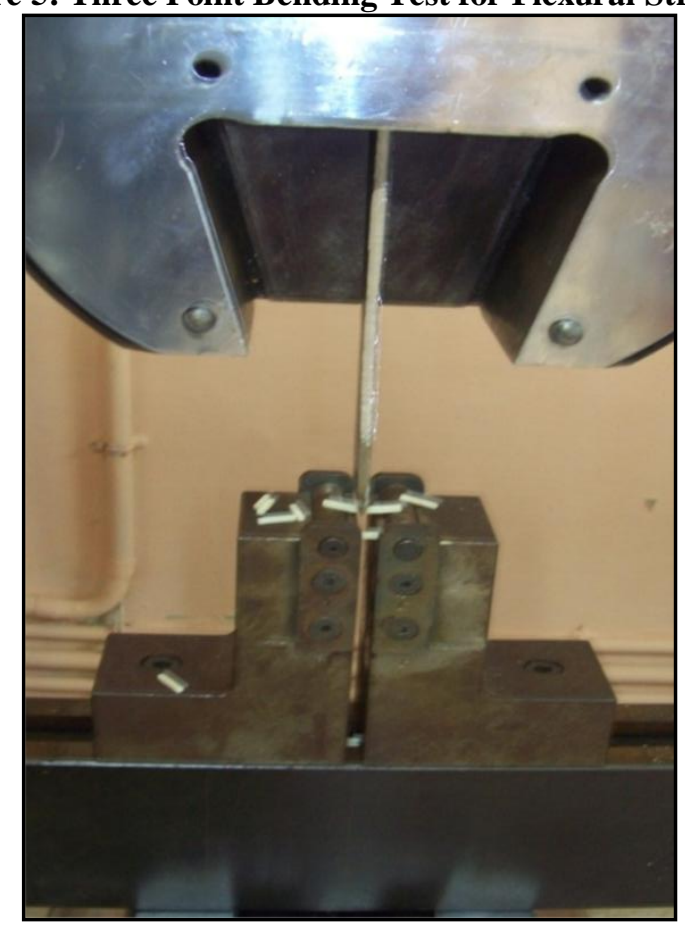

